\title{
Current topics in colorectal liver metastasis
}

\author{
Yoshiharu Sakai
}

Received: 30 July 2011/Published online: 11 August 2011

(C) Japan Society of Clinical Oncology 2011

Colorectal cancer is the second largest cause of cancer mortality in the United States [1], and the third largest cause in Japan [2]. At the time of diagnosis, $13 \%$ of patients will present with synchronous liver metastasis [3] and another $7.2 \%$ of patients with Stage I-III disease will develop metachronous liver metastasis even after a primary curative operation [4].

Improved surgical expertise and advances in chemotherapy combination regimens, such as FOLFOX and FOLFIRI, have contributed to profound improvement in outcomes in liver metastasis of colorectal cancer $[5,6]$. In order to obtain much better survival rates, several strategies combining surgery and new molecular targeted drugs, such as bevacizumab and cetuximab, have been investigated.

On the other hand, recent technological developments have provided much information regarding tumor biology, with tools to scrutinize cell-matrix interactions, cell-cell interactions, signal pathways, angiogenesis, cytokines, etc. [7]. In addition, an important role of immature myeloid cells in an early stage of metastasis has been reported [8]. Based on these findings of tumor biology, new molecular or cellular targeted drugs will be developed.

Here we present two current topics in colorectal liver metastasis: molecular mechanism of liver metastasis and treatment strategy for liver metastasis. The former focuses on several key roles of cytokines in liver metastasis, and the latter on aggressive resection combined with chemotherapy. We hope these review articles will lead to an understanding of current topics and facilitate research in this field.

Conflict of interest The author has no conflict of interest.

\section{References}

1. Jemal A, Tiwari RC, Murray T et al (2004) Cancer statistics. CA Cancer J Clin 54:8-29

2. Matsuda T, Marugame T, Kamo K et al (2008) Cancer incidence and incidence rates in Japan in 2002: based on data from 11 population-based cancer registries. Jpn J Clin Oncol 38:641-648

3. Japanese Society for Cancer of the Colon and Rectum (2011) Multi-Institutional Registry of large bowel cancer in Japan. Cases treated in 2000-2002, vol 29

4. Kobayashi H, Mochizuki H, Sugihara K et al (2007) Characteristics of recurrence and surveillance tools after curative resection for colorectal cancer. A multicenter study. Surgery 141:67-75

5. Kopetz S, Chang GJ, Overman MJ et al (2009) Improved survival in metastatic colorectal cancer is associated with adoption of hepatic resection and improved chemotherapy. J Clin Oncol 27:3677-3683

6. Gallagher DJ, Kemeny N (2010) Metastatic colorectal cancer: from improved survival to potential cure. Oncology 78:237-248

7. LeGolvan MP, Resnick M (2010) Pathobiology of colorectal cancer hepatic metastasis with an emphasis on prognostic factors. J Surg Oncol 102:898-908

8. Kitamura T, Fujishita T, Loetscher $P$ et al (2010) Inactivation of chemokine (C-C motif) receptor 1 (CCR1) suppresses colon cancer liver metastasis by blocking accumulation of immature myeloid cells in a mouse model. Proc Natl Acad Sci USA 107:13063-13068
Y. Sakai $(\bowtie)$

Department of Surgery, Kyoto University Graduate School

of Medicine, 54 Shogoin Kawara-cho, Sakyo-ku,

Kyoto 606-8507, Japan

e-mail: ysakai@kuhp.kyoto-u.ac.jp 